\title{
Quantum field-theoretical descriprion of neutrino oscilla- tions in T2K experiment
}

\author{
Vadim Egorov ${ }^{1,2, *}$ and Timofei Rusalev $^{2, * *}$ \\ ${ }^{1}$ Skobeltsyn Institute of Nuclear Physics, Lomonosov Moscow State University, 119991 Moscow, Rus- \\ sia \\ ${ }^{2}$ Faculty of Physics, Lomonosov Moscow State University, 119991 Moscow, Russia
}

\begin{abstract}
We consider neutrino oscillations in the T2K experiment using a new quantum field-theoretical approach to the description of processes passing at finite space-time intervals. It is based on the Feynman diagram technique in the coordinate representation, supplemented by modified rules of passing to the momentum representation. Effectively this leads to the Feynman propagators in the momentum representation being modified, while the rest of the Feynman rules remain unchanged. The approach does not make use of wave packets, the initial and final particle states are described by plane waves, which essentially simplifies the calculations. The oscillation fading out due to momentum distribution of the initial particles is taken into account. The obtained results reproduce the predictions of the standard description and confirm that the far detector position corresponds to the first minimum for muon production probability and the first maximum for electron production probability.
\end{abstract}

\section{Introduction}

Neutrino oscillations are a widely discussed and experimentally confirmed phenomenon, which is very important for particle physics. To describe this phenomenon either the quantum-mechanical approach in terms of plane waves or wave packets [1-3] or the quantum field-theoretical approach in terms of wave packets [3-5] are usually used. The plane wave QM description is not consistent since the production of states without definite masses violates energy-momentum conservation. This problem is circumvented in the framework of the QM and QFT descriptions in terms of wave packets, but the calculations of amplitudes and probabilities become very complicated. The reason is that the standard S-matrix theory is not convenient for describing processes, which take place at finite space-time intervals.

The idea of the novel approach is to adapt the standard S-matrix formalism for describing processes passing at finite space-time intervals by modifying the rules of transition from the coordinate representation to the momentum representation in the framework of the Feynman diagram technique, so that these rules reflect the experimental conditions. The approach, based on the work of Feynman [6], was put forward in the paper [7] and developed in the papers [8-11].

\footnotetext{
*e-mail: egorov@theory.sinp.msu.ru

**e-mail: rusalev.timofey@mail.ru
} 
Using this approach we describe processes, which can be investigated in the accelerator long baseline experiment T2K, where a neutrino flux is created by the decays of $\pi$ - and $K$ mesons. We consider processes, in which the neutrinos are detected due to scattering by nuclei of ${ }^{16} \mathrm{O}$, which in the simplified consideration by means of the impulse approximation may be reduced to the scattering at free neutrons, and by electrons.

\section{Description of neutrino oscillations}

\subsection{Quasielastic neutrino scattering by neutron}

We work in the framework of the minimal extension of the Standard Model by the right neutrino singlets. The charged-current interaction Lagrangian of the leptons takes the form

$$
L_{\mathrm{cc}}=-\frac{g}{2 \sqrt{2}}\left(\sum_{i, k=1}^{3} \bar{l}_{i} \gamma^{\mu}\left(1-\gamma^{5}\right) U_{i k} v_{k} W_{\mu}^{-}+\text {H.c. }\right),
$$

where $l_{i}$ is the field of the charged lepton of the $i$-th generation, $U_{i k}$ denotes the PMNS-matrix, and $v_{k}$ stands for the field of the neutrino state with definite mass $m_{k}$.

Let us consider the process, in which a neutrino is emitted by the decay of a $\pi$-meson and is detected in a reaction $v_{i}+{ }^{16} \mathrm{O} \rightarrow{ }^{15} \mathrm{O}+p+l^{-}$. Since the neutrino energy is much greater than the binding energy per nucleon, we use the impulse approximation, in which the scattering by a nucleus is represented as the sum of the scatterings by free nucleons. Therefore, we consider the quasielastic scattering of neutrinos by a neutron $v_{i}+n \rightarrow p+l^{-}$, where $l^{-}$ denotes an electron $e^{-}$or muon $\mu^{-}$. The process corresponds to the following diagram:

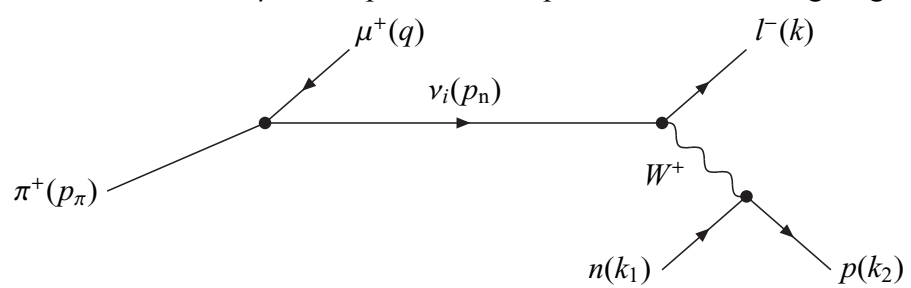

The four-momenta of the particles are designated as it is shown in the diagram. The neutrino $v_{i}$ is a virtual particle and is described by the propagator in the coordinate representation. The distance between the neutrino production and registration events is fixes and equals 295 $\mathrm{km}$. In this case, since the distance is macroscopically large, the virtual particle is almost on the mass shell due to GS-theorem, i.e. the relation $\left|p^{2}-m_{i}^{2}\right| \mid\left(p^{0}\right)^{2} \ll 1$ holds [4].

Following the prescription formulated in [7-11], we construct the so-called timedependent propagator of neutrino mass eigenstate $v_{i}$ in the momentum representation as the Fourier transform of the Feynman propagator $S_{i}^{\mathrm{c}}(z)$ of the fermion field in the coordinate representation, multiplied by the additional delta function $\delta\left(z^{0}-T\right)$, which fixes the time interval $T$ between the production and detection:

$$
S_{i}^{\mathrm{c}}(p, T)=\int \mathrm{d}^{4} z e^{i p z} S_{i}^{\mathrm{c}}(z) \delta\left(z^{0}-T\right) \approx i \frac{\hat{p}+m_{i}}{2 p^{0}} e^{-i \frac{m_{i}^{2}-p^{2}}{2 p^{0}} T},
$$

where $m_{i}$ is the mass of neutrino state $v_{i}$, and we have used the condition that the particle is close to the mass shell to derive the last expression.

For certainty, further we assume that the final charged lepton is a muon. Now we can use the modified propagator (2) to write out the amplitude of the process in the momentum 
representation for the case, when the time interval between the production and registration events equals $T$ :

$$
M=-i \frac{G_{\mathrm{F}}^{2}}{2 p_{\mathrm{n}}^{0}} \cos \theta_{\mathrm{C}} f_{\pi} m_{(\mu)} \sum_{i=1}^{3}\left|U_{2 i}\right|^{2} e^{-i \frac{m_{i}^{2}-p_{\mathrm{n}}^{2}}{2 p_{\mathrm{n}}^{0}} T} j_{\mu}\left(k_{1}, k_{2}\right) \bar{u}(k) \gamma^{\mu} \hat{p}_{\mathrm{n}}\left(1+\gamma^{5}\right) v(q),
$$

where $\theta_{\mathrm{C}}$ is the Cabibbo angle, $f_{\pi}$ is the pion decay constant of dimension of mass, $m_{(\mu)}$ is the muon mass, $j_{\mu}\left(k_{1}, k_{2}\right)=\left\langle p\left(k_{2}\right)\left|j_{\mu}^{(\mathrm{h})}\right| n\left(k_{1}\right)\right\rangle$ is the matrix element of the weak hadron current. Here and below we neglect the neutrino masses everywhere except in the exponential of the time-dependent propagator (2).

In our approximation, the squared modulus of the amplitude, averaged and summed over particles' polarizations (which is shown by the angle brackets), factorizes:

$$
\left\langle|M|^{2}\right\rangle=\left\langle\left|M_{1}\right|^{2}\right\rangle\left\langle\left|M_{2}\right|^{2}\right\rangle \frac{1}{4\left(p_{\mathrm{n}}^{0}\right)^{2}}\left[1-4 \sum_{\substack{i, k=1 \\ i<k}}^{3}\left|U_{2 i}\right|^{2}\left|U_{2 k}\right|^{2} \sin ^{2}\left(\frac{\Delta m_{i k}^{2}}{4 p_{\mathrm{n}}^{0}} T\right)\right],
$$

where $\left\langle\left|M_{1}\right|^{2}\right\rangle$ and $\left\langle\left|M_{2}\right|^{2}\right\rangle$ correspond to the pion decay and neutrino scattering by a neutron respectively, and the expression in the square brackets will be referred to as $P_{\mu \mu}\left(p_{\mathrm{n}}^{0}, T\right)$.

Let us find the probability of the process. The squared amplitude (4) is multiplied by the delta function of energy-momentum conservation $(2 \pi)^{4} \delta\left(p_{\pi}+k_{1}-q-k-k_{2}\right)$ and integrated with respect to the phase volume of the final particles. But the integration would result in variation of the virtual neutrino momentum direction, which contradicts the experimental setting. Thus, one must calculate the differential probability of the process, where $p_{\mathrm{n}}$ is fixed. It can be achieved by additionally multiplying the squared amplitude by $2 \pi \delta\left(p_{\mathrm{n}}-p\right)$, where $p$ is one specific value of $p_{\mathrm{n}}: p^{2}=0, \vec{p}$ is directed from the source to the detector and satisfies the conservation condition at the production vertex. It is equivalent to substituting $p$ instead of $p_{\mathrm{n}}$ in (4) and multiplying the result by $2 \pi \delta\left(p+q-p_{\pi}\right)$. When the intermediate virtual particle momentum is fixed, one can pass from the time interval $T$ to the distance $L$ according to the formula $T=L p^{0} /|\vec{p}|$. The differential probability has the form:

$$
\frac{\mathrm{d}^{3} W}{\mathrm{~d}^{3} p}=\frac{\mathrm{d}^{3} W_{1}}{\mathrm{~d}^{3} p} W_{2}^{(\mu)} P_{\mu \mu}(|\vec{p}|, L),
$$

where $\mathrm{d}^{3} W_{1} / \mathrm{d}^{3} p$ is the differential probability of pion decay, which produces neutrino with the fixed momentum $\vec{p}, W_{2}^{(\mu)}$ is the probability of neutrino scattering by a neutron with a muon in the final state, and the factor $P_{\mu \mu}(|\vec{p}|, L)$ is called, in the standard approach, the muon neutrino survival probability.

The additional delta function fixes not only the direction of neutrino momentum, but also its length, thus we must integrate the formula (5) with respect to $|\vec{p}|$. The final probability of detecting a muon in the considered process:

$$
\frac{\mathrm{d} W}{\mathrm{~d} \Omega}=\int \frac{\mathrm{d}^{3} W}{\mathrm{~d}^{3} p}|\vec{p}|^{2} \mathrm{~d}|\vec{p}|=\left.\frac{\mathrm{d} W_{1}}{\mathrm{~d} \Omega} W_{2}^{(\mu)}\right|_{|\vec{p}|=|\vec{p}|^{*}} P_{\mu \mu}\left(|\vec{p}|^{*}, L\right),
$$

where

$$
\frac{\mathrm{d} W_{1}}{\mathrm{~d} \Omega}=\frac{G_{\mathrm{F}}^{2} \cos ^{2} \theta_{\mathrm{C}} f_{\pi}^{2}}{8(2 \pi)^{2}} \frac{m_{(\mu)}^{2}\left(m_{\pi}^{2}-m_{(\mu)}^{2}\right)^{2}}{p_{\pi}^{0}\left(p_{\pi}^{0}-\left|\vec{p}_{\pi}\right| \cos \theta\right)^{2}}
$$


and the momentum $|\vec{p}|^{*}$ is determined by the energy-momentum conservation at the neutrino production vertex and has the following form:

$$
|\vec{p}|^{*}=\frac{m_{\pi}^{2}-m_{(\mu)}^{2}}{2\left(p_{\pi}^{0}-\left|\vec{p}_{\pi}\right| \cos \theta\right)},
$$

which implies that the coordinate system is chosen in such a way that the pion momentum $\vec{p}_{\pi}$ is directed along the $z$ axis, thus $\theta$ is the pion momentum polar angle.

The differential probability of neutrino production in the kaon decay $K^{+} \rightarrow v_{i}+\mu^{+}$is given by the same expression (7) with the replacements $\cos \theta_{\mathrm{C}} \rightarrow \sin \theta_{\mathrm{C}}, f_{\pi} \rightarrow f_{K}, m_{\pi} \rightarrow m_{K}$. The whole probability of finding an electron in the detection process differs from the obtained one (6) by the replacement $W_{2}^{(\mu)} \rightarrow W_{2}^{(e)}, P_{\mu \mu} \rightarrow P_{\mu e}$, where

$$
\begin{aligned}
P_{\mu e}(|\vec{p}|, L)= & -4 \sum_{\substack{i, k=1 \\
i>k}}^{3}\left[\operatorname{Re}\left(U_{1 i} U_{1 k}^{*} U_{2 i}^{*} U_{2 k}\right) \sin ^{2}\left(\frac{\Delta m_{i k}^{2}}{4|\vec{p}|} L\right)\right]+ \\
& +2 \sum_{\substack{i, k=1 \\
i>k}}^{3}\left[\operatorname{Im}\left(U_{1 i} U_{1 k}^{*} U_{2 i}^{*} U_{2 k}\right) \sin \left(\frac{\Delta m_{i k}^{2}}{2|\vec{p}|} L\right)\right]
\end{aligned}
$$

is called, in the standard approach, the $v_{\mu} \rightarrow v_{e}$ transition probability.

To obtain the probability of neutrino scattering by a neutron it is necessary to know the expression for the matrix element of the weak hadron current, which, due to the complex structure of hadrons, has to be parameterized by phenomenological form-factors. It was shown that the cross-section of neutrino scattering by a neutron has the following form [12]:

$$
\sigma_{(l)}=\frac{M^{2} G_{\mathrm{F}}^{2} \cos ^{2} \theta_{\mathrm{C}}}{8 \pi|\vec{p}|^{2}} \int_{Q_{\min }^{2}}^{Q_{\max }^{2}}\left(A_{(l)}\left(Q^{2}\right)-\frac{s-u}{M^{2}} B_{(l)}\left(Q^{2}\right)+\frac{(s-u)^{2}}{M^{4}} C_{(l)}\left(Q^{2}\right)\right) \mathrm{d} Q^{2},
$$

where $Q^{2}=-(p-k)^{2}$ is the transferred momentum, $s$ and $u$ are the Mandelstam variables, $M$ is the average mass of proton and neutron, index $l$ indicates the type of the final charged lepton, the coefficients $A_{(l)}, B_{(l)}, C_{(l)}$, being very bulky, can be found in the same paper [12]. In order to determine the limits of integration let us consider the domain of change of the Mandelstam variable $t=-Q^{2}$. Using the results obtained in book [13] and carrying out the calculations for our case, we obtain:

$$
Q_{\max }^{2}\left(m_{l}, p^{0}\right)=-m_{l}^{2}+\frac{p^{0}}{2 p^{0}+M}\left(m_{l}^{2}+2 p^{0} M \mp \sqrt{\left(m_{l}^{2}-2 p^{0} M\right)^{2}-4 m_{l}^{2} M^{2}}\right),
$$

where $m_{l}$ is the charged lepton mass.

\subsection{Neutrino scattering by an electron}

Let us consider the process of neutrino scattering by electrons, even though its contribution is significantly less than the previous one. The registration process goes through both the charged and neutral currents. The process is described by the following two diagrams: 

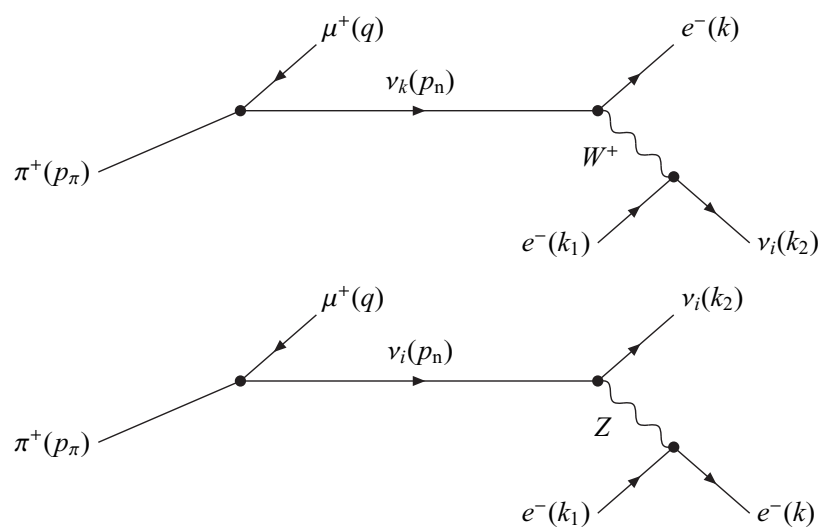

The four-momenta of the particles are designated as it is shown in the diagram.

The total amplitude of the process is as follows:

$$
\begin{aligned}
M_{\mathrm{tot}}^{(i)}= & -\frac{G_{\mathrm{F}}^{2}}{2 p_{\mathrm{n}}^{0}} \cos \theta_{\mathrm{C}} f_{\pi} m_{(\mu)} \bar{v}_{i}\left(k_{2}\right) \gamma^{\mu} \hat{p}_{\mathrm{n}}\left(1+\gamma^{5}\right) v(q) \times \\
& \times \bar{u}(k) \gamma_{\mu}\left[\left(B_{i}+A_{i}\left(-\frac{1}{2}+\sin ^{2} \theta_{\mathrm{W}}\right)\right)\left(1-\gamma^{5}\right)+A_{i} \sin ^{2} \theta_{\mathrm{W}}\left(1+\gamma^{5}\right)\right] u\left(k_{1}\right),
\end{aligned}
$$

where we have introduced the time-dependent factors:

$$
A_{i}=U_{2 i}^{*} e^{-i \frac{m_{i}^{2}-p_{\mathrm{n}}^{2}}{2 p_{\mathrm{n}}^{0}} T}, \quad B_{i}=U_{1 i}^{*} \sum_{k=1}^{3} U_{1 k} U_{2 k}^{*} e^{-i \frac{m_{k}^{2}-p_{\mathrm{n}}^{2}}{2 p_{\mathrm{n}}^{0}} T} .
$$

Performing the calculations, which are similar to those in the Subsection 2.1, we finally obtain the probability of the whole process:

$$
\frac{\mathrm{d} W}{\mathrm{~d} \Omega}=\int \sum_{i=1}^{3} \frac{\mathrm{d}^{3} W^{(i)}}{\mathrm{d}^{3} p}|\vec{p}|^{2} \mathrm{~d}|\vec{p}|=\left.\frac{\mathrm{d} W_{1}}{\mathrm{~d} \Omega} W_{2}\right|_{|\vec{p}|=|\vec{p}|^{*},}
$$

where the neutrino production probability $\mathrm{d} W_{1} / \mathrm{d} \Omega$ is defined by (7), and the momentum $|\vec{p}|^{*}$ is defined by (8). The neutrino detection probability $W_{2}$ has the standard form:

$$
W_{2}(|\vec{p}|, L)=P_{\mu e}(|\vec{p}|, L) W_{v_{e} e}+\left(1-P_{\mu e}(|\vec{p}|, L)\right) W_{v_{\mu} e},
$$

where $W_{v_{e} e}$ and $W_{v_{\mu} e}$ are the scattering probabilities of the corresponding neutrinos by an electron.

\section{Numerical integration}

Now we must average the obtained probabilities over the momentum distribution of the decaying particles, which produce neutrinos. Mesons ( $\pi$ - and $K-)$ in the decay volume have a distribution not only in the magnitude of the momentum, but also in the direction, so the angle between the source-detector line, which is at the angle $2.5^{\circ}$ to the $z$ axis, and the momentum of the decaying meson can vary. We introduce a coordinate system such that $\theta_{0}=2.5^{\circ}$, $\varphi_{0}=0$, and define the following unit vectors:

$$
\begin{aligned}
\vec{n}_{0} & =\left\{\sin \theta_{0}, 0, \cos \theta_{0}\right\} \text { - from the source to the detector, } \\
\vec{n} & =\left\{\sin \theta^{\prime} \cos \varphi^{\prime}, \sin \theta^{\prime} \sin \varphi^{\prime}, \cos \theta^{\prime}\right\} \text { - the meson momentum direction, }
\end{aligned}
$$


where $\theta^{\prime}$ is the angle between the $z$ axis and the randomly directed meson momentum. It is clear that the angle $\theta$ in formula (7), which is the angle between the initial meson momentum and the neutrino momentum, is the angle between these two unit vectors, which is easily found from their scalar product:

$$
\left(\vec{n}_{0} \vec{n}\right)=\left|\overrightarrow{n_{0}}\right||\vec{n}| \cos \theta=\cos \theta=\sin \theta_{0} \sin \theta^{\prime} \cos \varphi^{\prime}+\cos \theta_{0} \cos \theta^{\prime} .
$$

Let us consider the averaged probability for the case of the $\pi$-meson decay and the detection of lepton $l^{-}, l=\mu, e$ :

$$
\frac{\overline{\mathrm{d} W}}{\mathrm{~d} \Omega}=\int \mathrm{d} \varphi^{\prime} \sin \theta^{\prime} \mathrm{d} \theta^{\prime}\left|\vec{p}_{\pi}\right|^{2} \mathrm{~d}\left|\vec{p}_{\pi}\right| \rho_{\pi}\left(\left|\vec{p}_{\pi}\right|, \theta^{\prime}\right) \frac{\mathrm{d} W_{1}}{\mathrm{~d} \Omega}\left(\left|\vec{p}_{\pi}\right|, \theta\left(\theta^{\prime}, \varphi^{\prime}\right)\right) P_{\mu l}\left(|\vec{p}|^{*}, L\right) W_{2}^{(l)}\left(|\vec{p}|^{*}\right),
$$

where $\rho_{\pi}\left(\left|\vec{p}_{\pi}\right|, \theta^{\prime}\right)$ is the momentum distribution of the $\pi$-meson. Performing the change of variables $\left|\vec{p}_{\pi}\right| \rightarrow|\vec{p}|^{*}$, we arrive at the expression

$$
\overline{\frac{\mathrm{d} W}{\mathrm{~d} \Omega}}=\int \mathrm{d}|\vec{p}|^{*} \rho_{(v)}^{\pi}\left(|\vec{p}|^{*}\right) P_{\mu l}\left(|\vec{p}|^{*}, L\right) W_{2}^{(l)}\left(|\vec{p}|^{*}\right),
$$

where

$$
\rho_{(v)}^{\pi}\left(|\vec{p}|^{*}\right)=\int \mathrm{d} \varphi^{\prime} \sin \theta^{\prime} \mathrm{d} \theta^{\prime}\left|\vec{p}_{\pi}\right|^{2} \frac{D\left(\varphi^{\prime}, \theta^{\prime},\left|\vec{p}_{\pi}\right|\right)}{D\left(\varphi^{\prime}, \theta^{\prime}, \mid \vec{p}^{*}\right)} \rho_{\pi}\left(\left|\vec{p}_{\pi}\right|, \theta^{\prime}\right) \frac{\mathrm{d} W_{1}}{\mathrm{~d} \Omega}\left(\left|\vec{p}_{\pi}\right|, \theta\left(\theta^{\prime}, \varphi^{\prime}\right)\right)
$$

is the momentum distribution of the neutrinos produced in the pion decays. It is clear that the similar expression can be written for the neutrinos, which are produced in the decays of $K$-mesons. The total neutrino flux will be equal to the sum of the two distributions $\rho_{(v)}^{\pi}$ and $\rho_{(v)}^{K}$ with some weights $a_{\pi}$ and $a_{K}$ :

$$
\rho_{(v)}=a_{\pi} \rho_{(v)}^{\pi}+a_{K} \rho_{(v)}^{K},
$$

and the weights are related to each other, because we know the relative frequency of the decays of pions and kaons: 1 decayed kaon accounts for about 10 decayed pions, so $a_{\pi} / a_{K} \approx$ 10. Thus, the final probability with the neutrinos from both sources reads

$$
(\overline{\mathrm{d} W})_{\mathrm{d} \Omega}=\int \mathrm{d}|\vec{p}| \rho_{(v)}(|\vec{p}|) P_{\mu l}(|\vec{p}|, L) W_{2}^{(l)}(|\vec{p}|),
$$

where we denote $|\vec{p}|^{*}$ by $|\vec{p}|$ for short.

The form of neutrino flux dependence on the momentum for the angle $\theta_{0}=2.5^{\circ}$ is taken from the paper [14]. We fit this dependence with the following function:

$$
\rho_{(v)}(|\vec{p}|)= \begin{cases}a|\vec{p}|^{m}\left(\varepsilon+|\vec{p}|^{n}\right) e^{-(|\vec{p}|-b)^{2} / c}, & |\vec{p}|<0.65 \mathrm{GeV}, \\ y_{0}+A e^{-|\vec{p}| / z}, & |\vec{p}|>0.65 \mathrm{GeV} .\end{cases}
$$

The values of the constants are as follows: $a=87, m=0.16, \varepsilon=0.006, n=6.5, b=0.38$, $c=0.048, y_{0}=0.03, A=151, z=0.14$

The model parameters necessary to calculate the probability of neutrino oscillations are taken to be [15]: $\Delta m_{21}^{2}=7.39 \cdot 10^{-5} \mathrm{eV}^{2}, \Delta m_{32}^{2}=2.53 \cdot 10^{-3} \mathrm{eV}^{2}, \theta_{12}=0.5903, \theta_{23}=0.8657$, $\theta_{13}=0.1503, \delta_{\mathrm{CP}}=3.753$. 


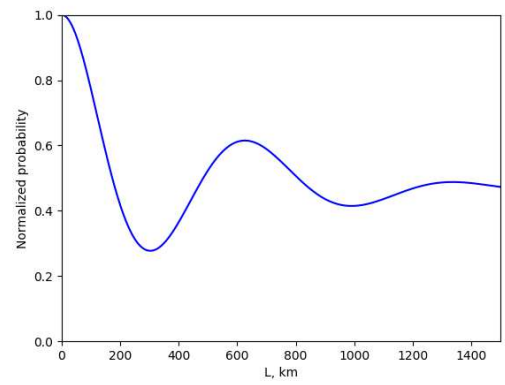

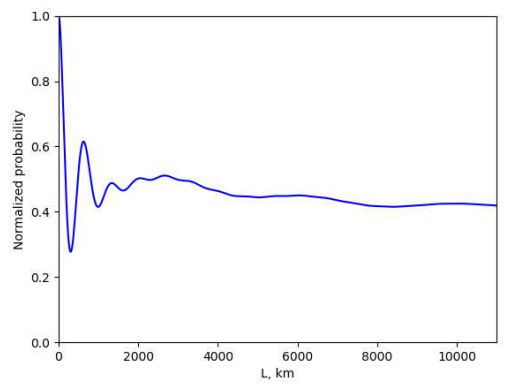

b

Figure 1. Normalized probability of the neutrino oscillation process with the detection by a nucleon and muon production on spatial scales a) $1500 \mathrm{~km}$ and b) $11000 \mathrm{~km}$

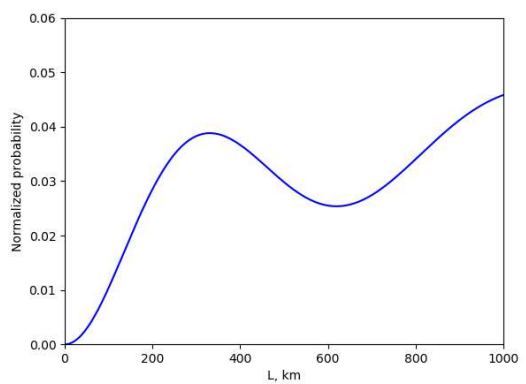

a

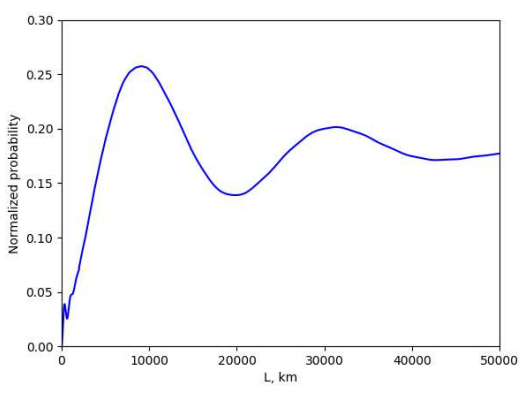

b

Figure 2. Normalized probability of the neutrino oscillation process with the detection by a nucleon and electron production on spatial scales a) $1000 \mathrm{~km}$ and b) $50000 \mathrm{~km}$

The results of numerical integration for the neutrino oscillation processes, where the neutrino is detected in the interaction with a neutron, producing a muon or an electron, are presented in Figs. 1, 2, respectively. The results of numerical integration for the neutrino oscillation process, where the neutrino is detected in the interaction with an electron are depicted in Figure 3. One can see that the detector position, $295 \mathrm{~km}$ in the T2K experiment, indeed corresponds to the first oscillation minimum for the muon production and the first oscillation maximum for the electron production.

\section{Conclusion}

The modified S-matrix approach in the framework of quantum field theory allows us to consistently obtain the expression for the probability of a neutrino oscillation process, which includes a process of virtual neutrino production in the source, its propagation over macroscopic distances and a registration process in the detector. In this approach, the initial and final particles are described by plane waves rather than wave packets, and the concept of flavor states is redundant. This formalism makes it possible to describe the neutrino oscillation 


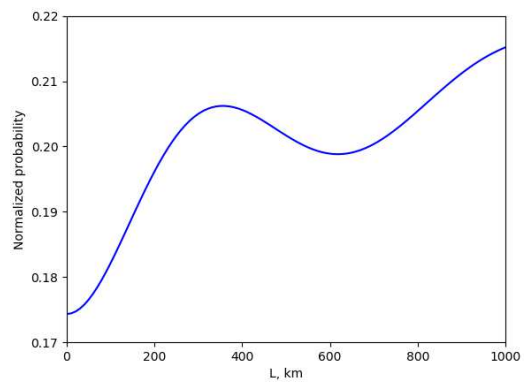

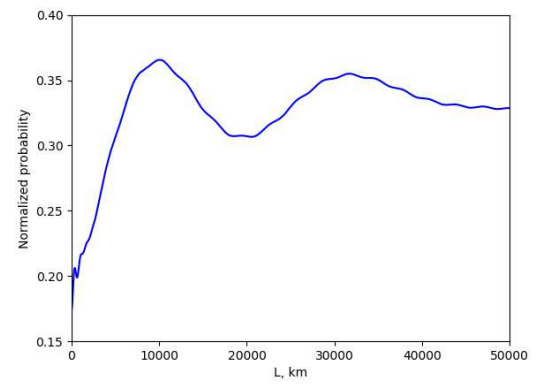

b

Figure 3. Normalized probability of the neutrino oscillation process with the detection by an electron on the spatial scales a) $1000 \mathrm{~km}$ and b) $50000 \mathrm{~km}$

experiments with the neutrinos propagating in the direction at an angle to the axis of the initial particle beam simply and naturally. The obtained results confirm that the position of the far detector in the $\mathrm{T} 2 \mathrm{~K}$ experiment corresponds to the first oscillation maximum for electron production processes and the first oscillation minimum for muon production processes. The developed formalism also allows one to describe the neutrino oscillation fading out, which arises due to the spread of momenta of decaying $\pi$ - and $K$-mesons.

\section{Acknowledgments}

The authors are grateful to I. Volobuev for useful discussions. The work of V. Egorov was supported by the Foundation for the Advancement of Theoretical Physics and Mathematics "BASIS".

\section{References}

[1] B.M. Pontecorvo, Sov. Phys. JETP 6, 429-431 (1957)

[2] C. Giunti and C.W. Kim, Fundamentals of Neutrino Physics and Astrophysics (Oxford University Press, UK, 2007) 247-254

[3] C. Giunti et al., Phys. Rev. D 48, 4310-4317 (1993)

[4] W. Grimus and P. Stockinger, Phys. Rev. D 54, 3414-3419 (1996)

[5] M. Beuthe, Phys. Rept. 375, 105-218 (2003)

[6] R.P. Feynman, Phys. Rev. 76, 769-789 (1949)

[7] I.P. Volobuev, Int. J. Mod. Phys. A 33, 1850075 (2018)

[8] V.O. Egorov and I.P. Volobuev, Phys. Rev. D 97, 093002 (2018)

[9] I.P. Volobuev and V.O. Egorov, J. Exp. Theor. Phys. 128, 713-719 (2019)

[10] I.P. Volobuev and V.O. Egorov, Theor. Math. Phys. 199, 562-576 (2019)

[11] V.O. Egorov and I.P. Volobuev, Phys. Rev. D 100, 033004 (2018)

[12] H. Gallagher et al., Ann. Rev. Nucl. Part. Sci. 61, 355-378 (2011)

[13] E. Byckling and K. Kajantie, Particle Kinematics (Wiley, 1973) 95-102

[14] K. Abe et al., Nucl. Instrum. Meth. A 659, 106-135 (2011)

[15] I. Esteban et al., JHEP 1901 106, (2019) 